\title{
Treatment options for type 2 diabetes mellitus in patients with chronic kidney disease - old and new drugs
}

\author{
Ana Ferreira, Filipa Bastos, Maria Carlos Cordeiro, Jorge Portugal \\ Endocrinology Department, Hospital Garcia de Orta, Almada, Portugal
}

\section{ABSTRACT}

Chronic kidney disease is highly prevalent in patients with diabetes mellitus. There are many specific aspects in the treatment of diabetes that have to be taken into account when there is concomitant nephropathy. All antihyperglycemic drugs can be used in earlier stages of chronic kidney disease. With worsening nephropathy, most will require dose adjustments (some eventually suspension) and increased monitoring of adverse events and kidney function. New treatment options that are safe and effective are now available for more advanced stages of disease. Moreover, findings from large clinical trials suggest that some drugs, namely GLP-1 receptor agonists and SGLT2 inhibitors, might potentially have kidney and cardiovascular protective effects, although clinical significance and putative mechanisms are not yet fully understood.

The aim of this review is to provide an updated overview of relevant data to guide clinical practice in the use of antihyperglycemic agents in chronic kidney disease patients, including older drugs and also the most recently available treatment options.

Keywords: type 2 diabetes mellitus, chronic kidney disease, cardiovascular outcomes, renal outcomes.

\section{INTRODUCTION}

Chronic kidney disease (CKD) is a frequent comorbidity in patients with diabetes mellitus (DM). Approximately $43 \%$ of individuals with type 2 DM concomitantly have CKD and, with advancing age, the prevalence is even higher (61\% of type 2 DM patients $\geq 65$ years $)^{1}$. Many drugs used to treat DM have some restrictions in these patients that have to be taken into account in clinical practice. New treatment options are emerging that are safe and eventually beneficial for CKD patients, namely, GLP-1 receptor agonists and SGLT2 inhibitors.

This review summarizes the most relevant evidence to guide clinical practice in using antihyperglycemic drugs, focusing on practical aspects, main risks and potential benefits in CKD (considering renal and cardiovascular outcomes, the last also being important in these high risk patients). Although all kinds of insulin can be used in every CKD stage effectively, an extensive description of this treatment option is beyond the scope of this review.

\section{BIGUANIDES}

The only biguanide currently available is metformin. This drug has multiple mechanisms of action, with the main a decrease in insulin resistance, especially in the liver, where it reduces glucogenolysis and neoglucogenesis, ultimately reducing the amount of glucose released in circulation. The insulin sensitizing action in muscle and fat tissues increases peripheral glucose uptake. Metformin also has an incretin-like effect, raising GLP-1 levels (which stimulate insulin secretion and decrease glucagon release) and promotes anaerobic glucose metabolism in the small intestine ${ }^{2}$. It is particularly effective in reducing fasting glucose levels (although it also has some effect in prandial levels) ${ }^{3}$, usually allowing $\mathrm{HbA} 1 \mathrm{c}$ reductions greater than $1 \%$, with very low hypoglycemia risk ${ }^{4}$. The most frequent adverse events include anorexia, nausea, abdominal pain, vomiting and diarrhea, which are dose-dependent and usually transient ${ }^{2}$, but might be particularly bothersome and reduce compliance. In order to prevent them, it is advisable to recommend taking the drug after a meal, start with a low dose (e.g. 500mg once or twice daily) and up-titrate slowly (e.g. weekly increases of $500 \mathrm{mg}$ until target dose achieved) ${ }^{2}$. Maximum recommended dose is $3000 \mathrm{mg} /$ day. Vitamin B12 deficiency (rarely leading to anemia) is also a concern when metformin is used chronically, so its levels must be checked regularly, as well as the full blood count ${ }^{2,5}$. Supplementation is warranted, if needed. The most serious adverse event related to biguanides is lactic acidosis, which is caused by respiratory chain inhibition that leads to a shift towards anaerobic metabolism and lactate production ${ }^{6}$. The evidence on this issue is conflicting, but in general, lactic acidosis seems to be a rare event ${ }^{6}$, particularly if metformin is adequately used (respecting contra-indications) ${ }^{2,6}$. There is some concern about an increased risk of lactic acidosis in CKD because metformin is excreted by the kidney ${ }^{3}$. In their systematic review, Inzuchi et al ${ }^{6}$ concluded that as long as kidney function is stable, proper monitoring is warranted, and dose adjustments are made as renal function deteriorates, the risk is low in mild and moderate CKD.

Metformin's dose has to be adjusted according to estimated glomerular filtration rate (eGFR). There is no absolute consensus on the best way of doing this, but it is generally accepted that it should be suspended if eGFR is below $30 \mathrm{ml} / \mathrm{min} / 1.73 \mathrm{~m}^{2}$. The American Diabetes Association joint position with the European Association for the Study of Diabetes (ADA/EASD) ${ }^{7}$ state that dose should be reduced in CKD 
stage 3B. The European Medicines Agency (EMA) states that for CKD stage $3 \mathrm{~A}$, maximum dose should be $2000 \mathrm{mg} /$ day, and for stage $3 \mathrm{~B}$, daily dose should not exceed $1000 \mathrm{mg} /$ day $^{8}$.

Metformin has long been regarded as dangerous in CKD patients, but it is now widely accepted as a safe option until further stages of the disease. In fact, it might even have renoprotective effects. Many studies using animal models (diabetic and non-diabetic nephropathies) and human cell lines have shown protective effects of this drug. The mechanisms described include decreased oxidative stress, anti-fibrotic actions with reduction of tubulointerstitial fibrosis and glomerulosclerosis, reduced podocyte loss, reduced albuminuria and prevention of vascular calcification ${ }^{9}$. Data available from human studies are scarce, mostly from observational, retrospective studies. Metformin has been associated to a lower risk of renal function decline or death when compared to sulfonylureas (independently of $\mathrm{HbA1c}$ over time) and a higher likelihood of graft survival and lower mortality in kidney transplant patients at 3 years post-transplant ${ }^{9}$. A recent post-hoc analysis from the TREAT (Trial to Reduce Cardiovascular Events with Aranesp (darbepoetin-alfa) Therapy) ${ }^{1}$, that included only patients with CKD (eGFR $20-60 \mathrm{ml} / \mathrm{min} / 1.73 \mathrm{~m}^{2}$ ), compared 508 metformin users with the same number of matched controls, and found that metformin users had lower rates of a kidney disease composite outcome including end-stage renal disease (ESRD) or death. However, no significant differences were found in creatinine doubling and progression to ESRD and the authors assumed that the benefit observed in the kidney composite outcome was primarily attributable to its effect on overall survival. This way, they conclude that metformin might not be able to prevent progression to ESRD after all. A possible explanation is that anti-fibrotic effects seen in animal models might only prevent progression of disease in earlier stages of $C K D^{1}$. Properly designed clinical trials to evaluate metformin's renoprotective effects in humans are still lacking.

Metformin may also reduce risk of cardiovascular events and death ${ }^{5}$, although data about this are still conflicting. The already mentioned post-hoc analysis from TREAT ${ }^{1}$ found that metformin users had lower rates of all-cause mortality, cardiovascular death and a cardiovascular disease composite including hospitalization for heart failure, myocardial infarction, stroke, myocardial ischemia or death, that remained after adjustment for several cardiovascular risk factors.

\section{SULFONYLUREAS}

Sulfonylureas exert their action by stimulating insulin secretion by the pancreatic beta cell (insulin secretagogues). They reduce fasting and prandial glucose levels and might be associated to $\mathrm{HbA1c}$ reductions greater than $1 \%{ }^{4}$. However, their effectiveness is usually not sustained over time 4,7 . The most common adverse event related to sulfonylureas is hypoglycemia, which is particularly a concern when long half-life sulfonylureas are used, especially glibenclamide (known in the USA as glyburide) ${ }^{7}$. Another common adverse event is weight gain 7 . Table I summarizes the main practical aspects of regularly used sulfonylureas.

The most important concern related to using these drugs in CKD patients is increasing hypoglycemia risk with deteriorating renal function $^{7}$. Gliclazide and glipizide are mostly metabolized in the liver to inactive metabolites, and only a minor part is excreted unaltered in urine ${ }^{12,14}$, so these are the safest sulfonylureas to use in these patients. Dose reduction might still be advisable as CKD progresses because there might be some increase in hypoglycemia risk. No specific recommendations exist on how to adjust dosing according to eGFR. There is no data on the use of gliclazide in patients with severe CKD, but considering its metabolism, as long as the dose is reduced, some recommend its use in these patients ${ }^{15}$, while others are more cautious and recommend against ${ }^{14}$. Glimepiride is also metabolized in the liver but some of its metabolites (that are subsequently excreted in urine) have hypoglycemic activity ${ }^{14,15}$, so when renal function deteriorates, hypoglycemia risk is significant. Dose reduction is recommended if eGFR falls below $60 \mathrm{ml} / \mathrm{min} / 1.73 \mathrm{~m}^{2}$ and it should be avoided under $30 \mathrm{ml} / \mathrm{min} / 1.73 \mathrm{~m}^{2} 15$. Again, there are no specific recommendations on dosing according to eGFR. Glyburide is metabolized in the liver and some metabolites are also active, equally excreted in the urine and bile ${ }^{15}$. Although biliary excretion might partially compensate for gradual loss of renal excretion in CKD, these patients are at high risk of hypoglycemia ${ }^{15}$. If eGFR is between 60 and $90 \mathrm{ml} / \mathrm{min} / 1.73 \mathrm{~m}^{2}$, patients should be treated with low dose and frequent monitoring. Glyburide should be suspended if eGFR falls below $60 \mathrm{ml} / \mathrm{min} / 1.73 \mathrm{~m}^{2} 15$.

There is no agreement on sulfonylureas' cardiovascular safety profile. Observational studies raised some concerns about this issue ${ }^{7}$ and it is still a matter of debate. Many observational studies, randomized control trials and meta-analysis have been performed, with inconsistent results ${ }^{16-18}$.

Evidence from the UK Prospective Diabetes Study and ADVANCE trials has confirmed the microvascular benefits of this drug class, namely, a reduction in the incidence or worsening of nephropathy, but it is still not known if these were related to the drug itself or to glucose-lowering effect ${ }^{16}$. They are generally regarded as neutral to the kidney ${ }^{5}$.

\section{Table I}

Sulfonylureas - dosage, route and adjustments in CKD patients. ${ }^{10-15}$

\begin{tabular}{c|c|c|c|c} 
Characteristic & Gliclazide & Glimepiride & Glipizide & Glyburide \\
\hline Dosage & MR: $30-120 \mathrm{mg} /$ day & $1-8 \mathrm{mg} /$ day & $2.5-40 \mathrm{mg} /$ day & $5-15 \mathrm{mg} /$ day \\
Dosing frequency & Once daily (MR) & Once daily & Once daily & Once daily \\
Route & Oral & Oral & Oral & Oral \\
CKD & ฟDose (?) & Dose if eGFR<60 & WDose (?) & Avoid if eGFR<60
\end{tabular}

MR - modified release. eGFR is expressed in $\mathrm{ml} / \mathrm{min} / 1.73 \mathrm{~m}^{2}$. 


\section{GLITAZONES}

Pioglitazone is the most frequently used drug in this class, and the only one available in our country. It acts through PPARy activation, which results in peripheral insulin sensitizing effect in the muscle, fat and liver ${ }^{19}$. It is effective in lowering mainly fasting glucose and can result in $\mathrm{HbA} 1 \mathrm{c}$ reductions greater than $1 \%^{4}$. Dosing varies between 15 and $45 \mathrm{mg}$, taken once daily, orally ${ }^{20}$. Hypoglycemia risk is low ${ }^{4}$. Pioglitazone has also a positive effect on lipid profile, raising HDL cholesterol, lowering triglycerides and improving LDL particle size (in spite of slightly raising their number $)^{4,19}$. This drug main problem is related to its adverse events. It can cause fluid retention and increased fat tissue (mainly subcutaneous), which translates into weight gain and peripheral edema, worsening heart failure symptoms ${ }^{4}$. This might be particularly bothersome for CKD patients that already have fluid retention. Another concern is the possibility of osteoporosis and increased risk of bone fracture, mainly in older women ${ }^{4}$. Elevated liver enzymes might also be seen and this should be monitored ${ }^{20}$. Probably the most worrisome adverse event that has been associated to pioglitazone is increased risk of bladder cancer, although the evidence supporting this association is inconsistent ${ }^{4}$. It is recommended not to prescribe this drug to patients with personal history of this kind of malignancy ${ }^{4}$.

Pioglitazone levels in blood are not raised in any stage of $\mathrm{CKD}^{20}$, so theoretically it could be used in all stages of the disease with the same dose regimen. However, it is advisable to reduce dose with progression of CKD, accounting for adverse events that might add to problems that come along with decreasing eGFR (e.g. fluid retention $)^{15}$. It is usually recommended not to exceed $15 \mathrm{mg} /$ day in these patients and avoid if on dialysis ${ }^{15,20}$.

Apart from the positive effect on the lipid profile, pioglitazone has been described to have other effects that might provide cardiovascular benefit going beyond glucose control. These include reducing blood pressure, plasma free fatty acids, inflammatory markers and procoagulant factors, slowing the progression of carotid intima-media thickness ${ }^{19}$. Several studies have evaluated the cardiovascular benefit from this drug. A recent meta-analysis of 9 randomized controlled trials (RCTs) including over 120100 patients with insulin resistance, pre-diabetes and type 2 DM was performed to evaluate this ${ }^{21}$. Among patients with pre-diabetes or insulin resistance, pioglitazone was associated with a significant lower risk of cardiovascular mortality, non-fatal myocardial infarction and non-fatal stroke - MACE (2 trials: RR $0.77 ; 95 \% \mathrm{Cl} 0.64$ to 0.93 ). Among patients with DM, pioglitazone was associated with a significant lower risk of MACE ( 5 trials: RR 0.83 ; $95 \% \mathrm{Cl} 0.72$ to 0.97$)$. However, this apparently positive cardiovascular effect was downsided by the fact that there was a significant increase in the risk of heart failure, bone fracture, edema and weight gain.

There is some evidence that pioglitazone might have beneficial effects on nephropathy. Qin et a ${ }^{22}$ performed a meta-analysis and review of 26 RCTs ( 9 with rosiglitazone and 17 with pioglitazone) including 8129 patients with type $2 \mathrm{DM}$. Overall, in patients with normo- and micro/macroalbuminuria, treatment with glitazones was associated with a significant decrease in urinary albumin excretion, but no improvement in eGFR was seen. It is not known if this is only related to glucose control or if other mechanisms are in place.

\section{DPP-4 INHIBITORS}

DPP-4 is an enzyme responsible for metabolizing and inactivating incretins (e.g. GLP-1, GIP), so its inhibitors increase glucose-dependent insulin secretion and reduce glucagon release. They are effective in lowering fasting and prandial glucose levels, with very low hypoglycemia risk. The major issue with these drugs is their limited efficacy, since they are usually associated with $\mathrm{HbA1c}$ reductions of less than $1 \%$. Their greatest advantage is their safety profile, with very low prevalence of side effects. The most important concern is the risk of acute pancreatitis ${ }^{5}$, but it is predictably rare.

The different DPP-4 inhibitors available and practical aspects of their use are described in Table II.

All DPP-4 inhibitors can be safely used in CKD. Linagliptin is the only one that does not need dose adjustment according to eGFR because it is metabolized in the liver and mainly excreted in bile, unlike the others that are cleared by the kidneys (table II). Some recommend saxagliptin should not be used in $\mathrm{ESRD}^{28}$, while others just recommend using a reduced dose $(2.5 \mathrm{mg} / \text { day })^{15}$.

DPP-4 inhibitors were the first antihyperglycemic drugs that had clinical trials specifically designed to evaluate cardiovascular safety (against placebo), as requested by regulatory authorities (TECOS 29 for sitagliptin, EXAMINE ${ }^{30}$ for alogliptin, SAVOR-TIMI $53^{31}$ for saxagliptin and CARMELINA ${ }^{32}$ for linagliptin). Vildagliptin has no cardiovascular outcome trial. These studies included only patients with established atherosclerotic vascular disease, except for SAVOR-TIMI 53 and CARMELINA that also included patients with cardiovascular

\section{Table II}

DPP-4 inhibitors - dosage, route and adjustments in CKD patients. ${ }^{23-27}$

\begin{tabular}{|c|c|c|c|c|c|}
\hline Characteristic & Sitagliptin & Vildagliptin & Linagliptin & Alogliptin & Saxagliptin \\
\hline Dosage & $100 \mathrm{mg}$ & $100 \mathrm{mg}$ & $5 \mathrm{mg}$ & $25 \mathrm{mg}$ & $5 \mathrm{mg}$ \\
\hline Dosing frequency & Once daily & $\begin{array}{l}\text { Twice daily } \\
\text { (50mg+50mg) }\end{array}$ & Once daily & Once daily & Once daily \\
\hline Route & Oral & Oral & Oral & Oral & Oral \\
\hline CKD & $\begin{array}{c}\text { eGFR } 30-50: 50 \mathrm{mg} / \text { day } \\
\text { eGFR <30: } 25 \mathrm{mg} / \text { day }\end{array}$ & eGFR<50: $50 \mathrm{mg} /$ day & $\begin{array}{c}\text { No dose } \\
\text { adjustment }\end{array}$ & $\begin{array}{c}\text { eGFR 30-50: } 12.5 \mathrm{mg} / \text { day } \\
\text { eGFR <30: } 6.25 \mathrm{mg} / \text { day }\end{array}$ & $\begin{array}{c}\text { eGFR<60: } 2.5 \mathrm{mg} / \text { day } \\
\text { ESRD - avoid (?) }\end{array}$ \\
\hline
\end{tabular}

eGFR is expressed in $\mathrm{ml} / \mathrm{min} / 1.73 \mathrm{~m}^{2}$ 
risk factors but no established disease. Primary outcome was the 3-point MACE already described (in TECOS, hospitalization for unstable angina was also included). All DPP-4 inhibitors demonstrated non-inferiority against placebo in primary outcome, but none was superior $29-32$. All-cause mortality was similar between groups in all studies $^{29-32}$. Saxagliptin was associated with increased risk of hospitalization for heart failure versus placebo (3.5\% vs. 2.8\%; HR 1.27; $\mathrm{P}=0.007)$, which is still not clear and has not been reproduced with other DPP-4 inhibitors $29,30,32$.

Evidence is emerging suggesting that these drugs may have renoprotective effects unrelated to their antihyperglycemic action $^{33,34}$. Several renal beneficial effects have been described in animal models of diabetic and non-diabetic nephropathy, human cell lines and even in human studies for all DPP-4 inhibitors available $^{34}$. These include anti-oxidative and anti-inflammatory actions, decreased podocyte loss, decreased albuminuria, decreased tubular damage, and anti-fibrotic actions with reduced glomerulosclerosis and interstitial fibrosis ${ }^{33,34}$. A detailed description of all molecular mechanisms potentially involved in these effects is beyond the scope of this review.

Cardiovascular outcome studies mentioned above also included some renal outcomes (secondary or evaluated on exploratory analysis).

In TECOS ${ }^{29,35}$, patients with eGFR $>30 \mathrm{ml} / \mathrm{min} / 1.73 \mathrm{~m}^{2}$ were included. There was a progressive eGFR decline along time in both groups, with slightly lower eGFR in the sitagliptin group, which persisted after adjustment for baseline eGFR, baseline $\mathrm{HbAlc}$ and change of $\mathrm{HbA1c}$ over time (overall estimated mean difference of $-1.43 \mathrm{ml} / \mathrm{min} / 1.73 \mathrm{~m}^{2}$; $95 \% \mathrm{Cl}-1.88$ to $-0.98 ; \mathrm{P}<0.0001)$. Sitagliptin marginally lowered urinary albumin/creatinine ratio (UACR) in a small subset of patients with available data on this parameter (overall mean difference of -0.18 $\mathrm{mg} / \mathrm{g} ; 95 \% \mathrm{Cl}-0.35$ to $-0.02 ; \mathrm{P}=0.031$ ). Overall, the authors conclude that sitagliptin has no significant impact on CKD outcomes irrespective of baseline eGFR.

Unlike TECOS, EXAMINE ${ }^{30}$ also included patients with eGFR $<30 \mathrm{ml} /$ $\mathrm{min} / 1.73 \mathrm{~m}^{2}$. No significant differences in eGFR change from baseline were found in any stage of CKD. The number of patients requiring dialysis was also similar between groups.

In SAVOR-TIMI 53 31,36 , patients with ESRD, previous renal transplant and creatinine $\geq 6.0 \mathrm{mg} / \mathrm{dL}$ were excluded. A composite renal outcome was considered (including doubling of creatinine level, initiation of dialysis, renal transplantation or creatinine $>6.0 \mathrm{mg} / \mathrm{dL}$ ), with a small non-significant difference against placebo $(2.2 \%$ for saxagliptin vs. $2.0 \%$ for placebo; HR $1.08 ; 95 \% \mathrm{Cl} 0.88$ to $1.32 ; \mathrm{P}=0.45$ ). Saxaglitpin reduced UACR in various levels of eGFR (at 2 years of follow-up, the difference in mean UACR change between saxagliptin and placebo groups was $-19.3 \mathrm{mg} / \mathrm{g}(\mathrm{P}=0.033)$ for eGFR $>50 \mathrm{~mL} /$ $\mathrm{min} / 1.73 \mathrm{~m}^{2} ;-105 \mathrm{mg} / \mathrm{g}(\mathrm{P}=0.011)$ for eGFR $30-50 \mathrm{~mL} / \mathrm{min} / 1.73 \mathrm{~m}^{2}$; and $-245.2 \mathrm{mg} / \mathrm{g}(\mathrm{P}=0.086)$ for eGFR $\left.<30 \mathrm{ml} / \mathrm{min} / 1.73 \mathrm{~m}^{2}\right)$. These findings had no correlation with $\mathrm{HbA1c}$ levels ${ }^{36}$. Saxagliptin was significantly superior to placebo in improving or avoiding UACR deterioration from baseline to end of trial for all baseline UACR categories (normo-, micro- and macroalbuminuria) ${ }^{36}$. There was no significant difference in eGFR change between saxagliptin and placebo arms, which was also observed in different UACR and eGFR categories ${ }^{36}$.

CARMELINA ${ }^{32}$ was the first of cardiovascular outcome studies also adequately powered to evaluate kidney outcomes and included a very large portion of patients with CKD (74\% of patients had CKD, 43\% with an eGFR below $45 \mathrm{~mL} / \mathrm{min} / 1.73 \mathrm{~m}^{2}$, and $15.2 \%$ with an eGFR below $30 \mathrm{~mL} / \mathrm{min} / 1.73 \mathrm{~m}^{2}$ ). ESRD patients were excluded. A secondary composite kidney outcome including first sustained ESRD, death due to renal failure or sustained decrease of $40 \%$ in eGFR from baseline was not significantly different between groups $(9.4 \%$ in linagliptin group vs. $8.8 \%$ in placebo group; HR $1.04 ; 95 \% \mathrm{Cl} 0.89$ to $1.22 ; \mathrm{P}=0.06$ ). However, linagliptin did have an apparent benefit in decreasing albuminuria progression (35.3\% of linagliptin patients vs. $38.5 \%$ of placebo patients; HR 0.86 ; $95 \% \mathrm{Cl} 0.78$ to $0.95 ; \mathrm{P}=0.003$ ).

In summary, DPP-4 inhibitors might have a beneficial effect on albuminuria progression, especially saxaglitpin and linagliptin. They were unable, however, to prevent kidney function deterioration in these trials.

These studies have some limitations. Median follow-up periods were short (18 months to 3.0 years) and renal outcomes were either secondary or evaluated on exploratory analysis, as already said. Furthermore, some effect from glucose control on outcomes cannot be fully excluded. Also, mainly patients with established atherosclerotic disease were included, so results cannot be extrapolated to lower risk scenarios. Another relevant aspect is that clinical significance of these findings remains to be fully clarified. Large, prospective, adequately powered and specifically designed for kidney outcome evaluation studies, as well as real world data, are necessary to improve our understanding about this issue.

\section{GLP-1 RECEPTOR AGONISTS}

GLP-1 receptor agonists act through several mechanisms, namely, increasing glucose-dependent insulin secretion and peripheral sensitivity, reducing glucagon release, delaying gastric empty, reducing appetite and increasing satiety ${ }^{37}$. They are divided into 2 different types - short and long-acting. The first include lixisenatide and exenatide (twice daily formulation); the second include liraglutide, dulaglutide, exenatide (weekly formulation), semaglutide and albiglutide. These drugs differ slightly in their properties ${ }^{38}$. Short-acting agonists tend to have a greater effect on delaying gastric emptying, having a more pronounced efficacy in reducing post-prandial glucose levels ${ }^{38}$. Long-acting do not significantly reduce gastric motility, exerting their action mainly through their effect on the pancreatic insulin and glucagon secretion ${ }^{38}$. The longer half-lives make long-acting GLP-1 receptor agonists more effective during the whole 24 hours, with a greater impact on fasting glucose levels ${ }^{38}$. Both increase satiety and reduce appetite, acting directly on the central nervous system ${ }^{38}$.

Long-acting GLP-1 receptor agonists are among the most effective drugs available to treat DM nowadays, able to produce $\mathrm{HbA} 1 \mathrm{c}$ reductions greater than $1 \%$ with very low hypoglycemia risk ${ }^{4}$. They are particularly beneficial for patients with excessive weight, because they can produce significant weight reductions ${ }^{5}$. 


\section{Table III}

GLP-1 receptor agonists - dosage, route and adjustments in CKD patients. ${ }^{38-44}$

\begin{tabular}{c|c||c||c|c|c|c|c|} 
Characteristic & Liraglutide & Exenatide* & Dulaglutide & Semaglutide & Albiglutide \\
\hline Dosage & 1.2 to $1.8 \mathrm{mg}$ & $2 \mathrm{mg}$ & 0.75 or $1.5 \mathrm{mg}$ & 0.5 or $1 \mathrm{mg}$ & 30 or $50 \mathrm{mg}$ \\
Dosing frequency & Once daily & Once weekly & Once weekly & Once weekly & Once weekly \\
Route & SC & SC & SC & SC & Once daily \\
CKD & Withdraw if ESRD & Withdraw if eGFR<50 & Withdraw if ESRD & Withdraw if ESRD & Withdraw if eGFR<30 & Withdraw if eGFR<30
\end{tabular}

SC - subcutaneous; * long-acting. eGFR is expressed in $\mathrm{ml} / \mathrm{min} / 1.73 \mathrm{~m}^{2}$.

The most relevant characteristics of GLP-1 receptor agonists available are in Table III.

The main disadvantage is that these drugs have to be administered subcutaneously, although some require weekly administration only. Also, some patients do not tolerate gastrointestinal side effects, namely, nausea, vomiting and diarrhea. Animal studies showed increased risk of medullary thyroid cancer ${ }^{4}$, but large studies have been conducted in humans and so far there have been no findings confirming a real malignancy risk ${ }^{45-49}$. However, these trials were not specifically designed to evaluate this and are not adequately powered to do it, so GLP-1 receptor agonists are still not recommended in patients with personal or family history of this disease 4 . Another concern is a possible increase in the risk of pancreatitis and pancreatic cancer $^{4}$. Evidence from large clinical trials ${ }^{45-48}$ does not seem to confirm this, with pancreatitis and pancreatic cancer not being significantly more frequent in treatment groups than in placebo arms. However, as for medullary thyroid cancer, these trials have not been powered to specifically address this issue, so the same recommendation applies.

GLP-1 receptor agonists do not require dose adjustment in CKD, as long as the patients still have an eGFR that allows their use. Liraglutide, dulaglutide and semaglutide are approved for use until eGFR $15 \mathrm{ml} / \mathrm{min} / 1.73 \mathrm{~m}^{2}$. In patients with ESRD, clinical experience is scarce, so withdrawing is recommended $39,41,42$. This gave physicians new effective options in these subjects, with very low hypoglycemia risk, unlike insulin and sulfonylureas, that had been until then the main drugs allowed in this setting. Gastrointestinal side effects might be the most limiting factor in CKD patients as they can lead to acute on chronic kidney injury. Exenatide and lixisenatide are renally excreted and have a more restricted recommended use in CKD ${ }^{40,44}$. Albiglutide is also eliminated by the kidney but differences in exposure to the drug are not clinically relevant. The recommendation not to use it if eGFR falls below $30 \mathrm{ml} / \mathrm{min} / 1.73 \mathrm{~m}^{2}$ is related to very little experience with its use in this setting ${ }^{43}$.

These drugs are also thought to potentially have renoprotective effects. They can reduce hyperfiltration that occurs in diabetic nephropathy through several glomerular and tubular actions, having a positive net effect on renal hemodynamics ${ }^{50}$.

Like DPP-4 inhibitors, GLP-1 receptor agonists were all tested for cardiovascular safety with specifically designed clinical trials against placebo ${ }^{45-49}$. All trials included mostly (or only) patients with established atherosclerotic cardiovascular disease, with the exception of dulaglutide's trial (REWIND), and demonstrated non-inferiority against placebo in the 3-point MACE (lixisenatide also included hospitalization for unstable angina in primary outcome $)^{45-49}$. Surprisingly, liraglutide, semaglutide and albiglutide have showed apparent cardiovascular benefit with superiority in the 3-point MACE, with relative risk reductions of $13 \%, 26 \%$ and $22 \%$, respectively. Absolute differences between groups were $1.9 \%, 2.3 \%$ and $2 \%$, respectively. When analyzing 3-point MACE elements separately in these 3 trials, it becomes clear that MACE reduction with liraglutide was mostly attributable to cardiovascular mortality reduction, with semaglutide was mostly driven by reduction in non-fatal stroke and with albiglutide the only significantly reduced endpoint was myocardial infarction.

These trials also included renal outcomes in their analysis (as secondary endpoints).

LEADER trial (with liraglutide) ${ }^{45}$ included patients with CKD, mostly with eGFR $>30 \mathrm{ml} / \mathrm{min} / 1.73 \mathrm{~m}^{2}$. Along the trial, eGFR declined progressively, slightly less in the liraglutide group $(-2 \% ; P=0.01)^{51}$. A composite renal outcome including new-onset albuminuria, persistent doubling of creatinine and eGFR $<45 \mathrm{ml} / \mathrm{min} / 1.73 \mathrm{~m}^{2}$, need for continuous replacement therapy and death due to renal disease, was significantly less frequent in the liraglutide group $(5.7 \%$ vs. $7.2 \% ; \mathrm{HR} 0.78 ; 95 \% \mathrm{Cl}$ 0.77 to $0.92 ; \mathrm{P}=0.003)$. This was mainly due to decreased frequency of new-onset macroalbuminuria (3.4\% vs. $4.6 \%$; $\mathrm{HR} 0.74 ; 95 \% \mathrm{Cl} 0.60$ to $0.91 ; \mathrm{P}=0.004)$. Separately, none of the other endpoints had significant differences. New-onset microalbuminuria also occurred in fewer patients in the liraglutide group (49.1\% vs. 53.5\%; HR 0.87; $95 \% \mathrm{Cl} 0.83$ to $0.93 ; \mathrm{P}<0.001)$.

Exenatide's cardiovascular safety was evaluated in EXSCEL trial ${ }^{46}$. Two renal composite outcomes were also studied ${ }^{52}$ : the first included $40 \%$ eGFR decline, renal replacement and renal death and the second included the first plus new-onset macroalbuminuria. There were no significant differences between groups in the first, but the second was slightly less frequent in the exenatide group, with statistical significance only after adjusting the HR for age, sex, region, ethnicity, duration of diabetes, prior history of CV event, insulin use, baseline $\mathrm{HbA} 1 \mathrm{c}$, eGFR and BMI (5.8\% vs. $6.5 \%$; adjusted $\mathrm{HR} 0.85,95 \% \mathrm{Cl} 0.73$ to $0.98 ; P=0.027)$. No significant differences in eGFR were found. Given this finding, it would be fair to assume that exenatide could have a positive effect on new-onset macroalbuminuria, but when analyzed separately, although it was less frequent in the exenatide group, the difference was not statistically significant ( 2.2 vs. $2.5 \%$; $P=0.19$ ). This way, potential renal benefits from this drug remain unclear.

Semaglutide's cardiovascular outcomes were studied in SUSTAIN-6 trial $^{47}$. Patients in all stages of CKD were included. New-onset or worsening nephropathy (including persistent macroalbuminuria, persisting 
doubling of creatinine levels and an eGFR $<45 \mathrm{ml} / \mathrm{min} / 1.73 \mathrm{~m}^{2}$ or the need for continuous renal replacement therapy) occurred less frequently with semaglutide (3.8\% vs. $6.1 \%$; $\mathrm{HR} 0.64 ; 95 \% \mathrm{Cl} 0.46$ to 0.88 ; $\mathrm{P}=0.005)$.

The most recently available results come from the HARMONY trial $^{48}$, evaluating albiglutide's cardiovascular safety. Renal function (eGFR) was not significantly different between groups. No other renal outcomes were tested.

The ELIXA trial (with lixisenatide) ${ }^{49}$ included patients with CKD, none with ESRD. They documented percentual change in the UACR ratio from baseline to 108 weeks, showing a modest difference in favor of lixisenatide over placebo ( $24 \%$ vs. $34 \%, P=0.004)$, but this difference was attenuated after adjustment for $\mathrm{HbA1c}(\mathrm{P}=0.07)$.

Dulaglutide's cardiovascular outcome trial (REWIND) has ended, but results are still unpublished. This trial has two important features that make it different from others: first, the study population has a lower baseline cardiovascular risk, with only around $30 \%$ of the patients having established cardiovascular disease (against the 73 to $100 \%$ found in the already mentioned trials); second, the median follow-up is longer ( $>5$ years). Preliminary results have become public and dulaglutide demonstrated superiority in reducing 3-point MACE, although there are no data on the extent of this effect ${ }^{53}$.

Dulaglutide's renal outcomes were studied in the AWARD-7 clinical trial ${ }^{54}$, compared to insulin glargine. A secondary outcome from this trial included change in eGFR and UACR. Patients in all stages of CKD were included. At 26 and 52 weeks, eGFR did not change in the dulaglutide group, but declined from baseline with insulin glargine. At 52 weeks, eGFR was significantly higher with dulaglutide than with insulin glargine. In patients with macroalbuminuria, dulaglutide reduced its progression more significantly than insulin.

Most of these trials included a majority of patients with established atherosclerotic disease, so it is still not clear whether the results apply in lower risk populations. Follow-up periods were relatively short which makes long-term extrapolation of data not possible. Differences were found between studies, so it remains unclear if the potential benefits are a class effect. Moreover, although statistically significant, the absolute risk differences found were of small magnitude and clinical significance still has to be clarified. Considering these trials' design, it is difficult to ascertain the extent of the contribution from many factors to the final outcome, namely, direct drug effect, glucose control and other medications. Renal outcomes were mainly included as secondary or evaluated in exploratory post-hoc analysis, and not all trials were powered to study individual kidney outcomes. In spite of all these limitations, the evidence available so far is in favor of some GLP-1 receptor agonists having potential beneficial cardiovascular and renal effects and this has been reflected on the ADA/EASD guidelines released in 2018.

\section{SGLT2 INHIBITORS}

SGLT2 inhibitors exert their action by markedly reducing proximal tubular glucose reabsorption in the kidney, inducing glucosuria ${ }^{55}$.
This transporter is responsible for approximately $90 \%$ of glucose reabsorption in the nephron, with the remaining $10 \%$ reabsorbed by SGLT1 ${ }^{55}$. There are four drugs approved in this class - dapagliflozin, empagliflozin, canagliflozin and ertugliflozin. Others are still in development. Canagliflozin also inhibits SLGT1, which is important for intestinal glucose absorption. SGLT2 inhibitors have been associated with $\mathrm{HbA} 1 \mathrm{c}$ reductions greater than $1 \%$, reducing fasting and prandial glucose levels, with very low hypoglycemia risk ${ }^{4}$. Modest weight loss is also a possible effect ${ }^{4}$. The most frequent adverse events include genital infections and increased urinary output ${ }^{4}$. Less commonly, urinary tract infections and dehydration (with all its possible consequences) might also happen ${ }^{4}$. It is advisable to recommend increasing fluid intake and, if possible, avoid simultaneous treatment with loop diuretics or, if used concomitantly, monitor fluid status and kidney function closely ${ }^{56-59}$. Another adverse event that can be particularly serious is euglycemic ketoacidosis ${ }^{4}$. Patients at greatest risk are type $1 \mathrm{DM}$, but it has also been described in type $2 \mathrm{DM}^{4,56-59}$. Type $2 \mathrm{DM}$ patients that are thought to be at the greatest risk are the ones with low beta cell reserve ${ }^{56-59}$. It is important for patients to be aware of the symptoms and be instructed on how to proceed ${ }^{4}$. Another concern about SGLT2 inhibitors is a possible increase in bone fracture risk. A recent systematic review and meta-analysis including a total of 40 RCTs and 32343 type 2 DM patients found no association between SGLT2 inhibitors' use and fracture risk $^{60}$. Lastly, canagliflozin was associated with an increase in lower limb amputation risk in the CANVAS trial (its cardiovascular safety trial). This was not seen with other SGLT2 inhibitors so far and has not been confirmed in a very large real life study - OBSERVE-4D ${ }^{61}$. In this study, neither canagliflozin nor dapagliflozin or empagliflozin showed a consistent increased risk of below the knee limb amputations comparing to non-SGLT2 inhibitors in the overall population or within the subpopulation of patients with established cardiovascular disease. However, the authors do recognize that none of the analyses performed was sufficiently powered to rule out the possibility of a modest effect.

Considering their mechanism of action, SGLT2 inhibitors' efficacy declines as eGFR falls below $60 \mathrm{ml} / \mathrm{min} / 1.73 \mathrm{~m}^{2}$. Accordingly, dose reductions are generally recommended (table IV) and if the patient already has an eGFR below $60 \mathrm{ml} / \mathrm{min} / 1.73 \mathrm{~m}^{2}$, these drugs should not be started ${ }^{62}$.

Cardiovascular safety trials for SGLT2 inhibitors also had positive results with empagliflozin and canagliflozin 63,64 showing superiority in the 3-point MACE against placebo, with a relative risk reduction of $14 \%$ in both, in a population of high cardiovascular risk (mainly with established atherosclerotic cardiovascular disease). Absolute differences were $1.6 \%$ and 4.6 participants per 1000 patient-years, respectively. When analyzing individual endpoints, there were no significant differences in non-fatal myocardial infarction and stroke with empagliflozin, only in cardiovascular mortality. With canagliflozin, no statistically significant differences were found when cardiovascular death, non-fatal myocardial infarction and stroke were analyzed separately. Dapagliflozin ${ }^{65}$ demonstrated non-inferiority in 3-point MACE, but was not superior. Ertugliflozin's cardiovascular outcome study is still ongoing (VERTIS-CV).

Renal outcomes were also included in these trials. 
Table IV

SGLT2 inhibitors - dosage, route and adjustments in CKD patients. ${ }^{56-59}$

\begin{tabular}{c||c|c|c}
\multicolumn{1}{c|}{ Characteristic } & Dapagliflozin & Empagliflozin & Canagliflozin \\
\hline Dosage & 5 or $10 \mathrm{mg}$ & 10 or $25 \mathrm{mg}$ & 100 or $300 \mathrm{mg}$ \\
Dosing frequency & Once daily & Once daily & Once daily \\
Route & Oral & Oral & Oral \\
CKD & eGFR<60: withdraw & eGFR $45-60: 10 \mathrm{mg}$ & eGFR $45-60: 100 \mathrm{mg}$ \\
& & eGFR<45: withdraw & Oral \\
eGFR is expressed in $\mathrm{ml} / \mathrm{min} / 1.73 \mathrm{~m}^{2}$. & & & eGFR<45: withdraw \\
\end{tabular}

EMPA-REG OUTCOME trial (with empagliflozin) ${ }^{63}$ was the first to have results available. It included CKD patients with eGFR $>30 \mathrm{ml} /$ $\mathrm{min} / 1.73 \mathrm{~m}^{2}$. Renal outcomes were prespecified as secondary and a post-hoc analysis was also performed. Empagliflozin was found to have significantly less incident or worsening nephropathy against placebo ( $12.7 \%$ vs. $18.8 \%$; HR 0.61; $95 \% \mathrm{Cl} 0.53$ to $0.70 ; \mathrm{P}<0.001$ ), doubling serum creatinine level associated with eGFR $\leq 45 \mathrm{ml} / \mathrm{min} / 1.73 \mathrm{~m}^{2}(1.5 \%$ vs. $2.6 \%$; $\mathrm{HR} 0.56 ; 95 \% \mathrm{Cl} 0.39$ to $0.79 ; \mathrm{P}<0.001)$, initiation of renal replacement therapy $(0.3 \%$ vs. $0.6 \%$; $\mathrm{HR} 0.45 ; 95 \% \mathrm{Cl} 0.21$ to 0.97 ; $\mathrm{P}=0.04)$ and progression to macroalbuminuria (11.2\% vs. $16.2 \%$; HR $0.62 ; 95 \% \mathrm{Cl} 0.54$ to $0.72 ; \mathrm{P}<0.001)$. No significant differences were found in new-onset albuminuria in patients with previously normal UACR ( $51.5 \%$ vs $51.2 \%$; HR $0.95 ; 95 \% \mathrm{Cl} 0.87$ to $1.04 ; \mathrm{P}=0.25$ ). In the first month of treatment, there was a slight decrease in the eGFR in the empagliflozin group. Thereafter, eGFR remained stable in the empagliflozin group and declined steadily in the placebo arm.

The CANVAS program included 2 trials evaluating canagliflozin cardiovascular and renal outcomes (CANVAS and CANVAS-R) ${ }^{64}$. Patients with eGFR $>30 \mathrm{ml} / \mathrm{min} / 1.73 \mathrm{~m}^{2}$ were included. Albuminuria progression (defined as $30 \%$ increase or progression from normo to micro/macroalbuminuria or progression from micro to macroalbuminuria) was less frequent with canagliflozin (HR 0.73; $95 \% \mathrm{Cl} 0.67$ to 0.79). Albuminuria regression was also more frequent with this drug. Canagliflozin also performed better in the composite outcome of sustained $40 \%$ reduction in eGFR, need for renal-replacement therapy, or death from renal causes (HR $0.60 ; 95 \% \mathrm{Cl} 0.47$ to 0.77 ). Annual eGFR decline was slower and mean UACR was lower in participants treated with canagliflozin. A specific trial was designed to evaluate potential benefits of canagliflozin in patients with type $2 \mathrm{DM}$ and CKD (eGFR between 30 and $90 \mathrm{ml} / \mathrm{min} / 1.73 \mathrm{~m}^{2}$ and macroalbuminuria, all treated with renin-angiotensin system blockade) - CREDENCE ${ }^{66}$. Efficacy and safety of canagliflozin $100 \mathrm{mg}$ /day in delaying diabetic nephropathy progression was evaluated. It was stopped earlier due to achievement of pre-specified efficacy criteria. Primary outcome was a composite of ESRD (dialysis for at least 30 days, kidney transplantation, or an estimated eGFR of $<15 \mathrm{ml} / \mathrm{min} / 1.73 \mathrm{~m}^{2}$ sustained for at least 30 days), doubling of the serum creatinine level from baseline sustained for at least 30 days, or death from renal or cardiovascular disease. There was a $30 \%$ relative risk reduction in this outcome with canagliflozin ( $\mathrm{HR} 0.70 ; 95 \% \mathrm{Cl} 0.59$ to $0.82 ; \mathrm{P}=0.00001$ ), with an absolute difference of 43.2 vs. 61.2 events/1000 patient-years. This was mainly driven by doubling of serum creatinine and ESRD. The geometric mean of the UACR was lower by $31 \%$ on average during follow-up and eGFR decline was slower in the canagliflozin group $(-1.85 \pm 0.13$ in the canagliflozin group vs. $-4.59 \pm 0.14 \mathrm{ml} / \mathrm{min} / 1.73 \mathrm{~m}^{2}$ in placebo group, per year). Adverse events were similar between groups, including lower limb amputations.

Dapagliflozin was studied in the DECLARE-TIMI 58 trial $^{65}$. This was somehow different because it included only $40 \%$ of patients with already established atherosclerotic cardiovascular disease (the remaining only with cardiovascular risk factors) and patients with eGFR $<60 \mathrm{ml} /$ $\mathrm{min} / 1.73 \mathrm{~m}^{2}$ at screening were excluded (a few ended up being included because eGFR was lower at randomization). A renal composite endpoint including sustained $40 \%$ reduction in eGFR, need for renal-replacement therapy, or death from renal or cardiovascular causes was evaluated. In the overall population, the incidence was significantly lower in the dapagliflozin group (4.3\% vs. $5.6 \%$; HR $0.76 ; 95 \% \mathrm{Cl} 0.67$ to 0.87$)$. The difference still favored dapagliflozin after exclusion of cardiovascular causes for death from this composite endpoint (1.5\% vs. $2.8 \%$; $\mathrm{HR} 0.53 ; 95 \% \mathrm{Cl} 0.43$ to 0.66 ). As for canagliflozin, a specific trial was designed to evaluate efficacy and safety of dapagliflozin $10 \mathrm{mg} /$ day in delaying diabetic and non-diabetic nephropathy progression - DAPA-CKD. It is currently ongoing.

Renal outcomes were also included in trials designed to study the use of SGLT2 inhibitors in heart failure-EMPEROR-Reduced, EMPEROR-Preserved and DAPA-HF. Results are not available yet ${ }^{67}$.

Considering all these data, SGLT2 inhibitors seem to have the most promising renal benefit seen so far. Possible renoprotective mechanisms include decreased intraglomerular pressure (tubuloglomerular feedback effects), albuminuria reduction, decrease of pro-inflammatory and pro-fibrotic pathways, improving oxygenation of tubular cells, improving endothelial dysfunction, reducing blood pressure and improving cardiac function optimizing renal perfusion ${ }^{67}$.

All the evidence coming from these large trials faces very similar limitations to those already described for GLP-1 receptor agonists. Specific trials to evaluate renal outcomes are most likely going to elucidate physicians as to the apparent benefit that this class seems to have in delaying/preventing nephropathy progression, and some are already ongoing.

\section{CONCLUSION}

In October 2018, ADA and EASD updated their joint guidelines on diabetes $\mathrm{care}^{7}$, making what was probably the greatest change in the last years, regarding new evidence available from the above described cardiovascular safety trials. Metformin remains the first drug of choice 
(unless contra-indicated or not tolerated), but when considering other options (as alternatives or in addition to metformin), they recommend considering the presence of atherosclerotic cardiovascular disease. If present, then patients are to be divided into 2 groups: predominant atherosclerotic cardiovascular disease and predominant heart failure or CKD. The main gold is to choose, in the first place, drugs that have proven cardiovascular benefit in clinical trials, that are among GLP-1 receptor agonists and SGLT2 inhibitors. If atherosclerotic cardiovascular disease is predominant, both are reasonable options. If heart failure or CKD are more significant, then SGLT2 inhibitors, if still useful considering eGFR, should be the drugs of choice. In what concerns CKD patients, this choice is justified because SGLT2 inhibitors demonstrated a more pronounced and consistent beneficial effect on renal outcomes evaluated in cardiovascular safety trials, namely, nephropathy and albuminuria progression. Discussion of the rationale for prescribing SGLT2 inhibitors in heart failure patients as well as an extensive description of other aspects of the guidelines goes beyond the scope of this review.

In conclusion, diabetes care has suffered several changes in the last years. New drugs have become widely available and provided robust evidence of safety and efficacy. Some might even have benefits that extend beyond glucose control, with potential kidney and cardiovascular protective effects. The clinical significance of clinical trials' findings is likely to become clearer in the future with real life data and larger periods of follow-up. It is, however, important not to forget that older drugs might also be good treatment options for many CKD patients, as long as used carefully and appropriately.

\section{Disclosure of potential conflicts of interest: none declared.}

\section{References}

1. Charytan DM, Solomon SD, Ivanovich P, Remuzzi G, Cooper ME et al. Metformin use and cardiovascular events in patients with type 2 diabetes and chronic kidney disease. Diabetes Obes Metab 2019; $1-10$

2. Silva-Nunes J. Practical aspects on the therapy with metformin. Revista Portuguesa de Diabetes 2014; 9(3): 127-132

3. Metformin - European Medicines Agency summary of product characteristics. Accessed on March 2019

4. Polonsky K and Burant CF. Type 2 Diabetes Mellitus - Management. In: Williams textbook of Endocrinology 13th ed. Elsevier, 2016: 1418-1436

5. American Diabetes Association Standards of Medical Care in Diabetes - 2019. Diabetes care. 2019; $42(1)$

6. Inzucchi SE, Lipska KJ, Mayo H, Bailey CJ and McGuire DK. Metformin in patients with type 2 diabetes and kidney disease: a systematic review. JAMA 2014; 312(24): 2668-75

7. Davies MJ, D'Alessio DA, Fradkin J, Kernan WK, Mathieu C et al. Management of hyperglycaemia in type 2 diabetes, 2018. A consensus report by the American Diabetes Association (ADA) and the European Association for the Study of Diabetes (EASD). Diabetes Care 2018; 41(12): 2669-2701

8. European Medicines Agency's Committee for Medicinal Products for Human Use. Assessment Report - Metformin containing medicinal products. EMA/867221/2016

9. De Broe ME, Kajbaf F and Lalau JD. Renoprotective Effects of Metformin. Nephron 2018; 138(4): 261-274

10. Gliclazide - European Medicines Agency summary of product characteristics. Accessed on March 2019

11. Glimepiride - European Medicines Agency summary of product characteristics. Accessed on March 2019

12. Glipizide - European Medicines Agency summary of product characteristics. Accessed on March 2019

13. Glibenclamide - European Medicines Agency summary of product characteristics. Accessed on March 2019

14. Betônico CC, Titan SM, Correa-Giannella ML, Nery M and Queiroz M. Management of diabetes mellitus in individuals with chronic kidney disease: therapeutic perspectives and glycemic control. Clinics (Sao Paulo) 2016; 71(1): 47-53

15. Ioannidis I. Diabetes treatment in patients with renal disease: Is the landscape clear enough? World J Diabetes 2014; 5(5): 651-8
16. Khunti K, Chatterjee S, Gerstein HC, Zoungas S and Davies MJ. Do sulphonylureas still have a place in clinical practice? Lancet Diabetes Endocrinol 2018; 6(10): 821-832

17. Azoulay $L$ and Suissa S. Sulfonylureas and the risks of cardiovascular events and death: a methodological meta-regression analysis of the observational studies. Diabetes Care 2017; 40(5): 706-714

18. O'Brien MJ, Karam SL, Wallia A, Kang RH, Cooper AJ et al. Association of second-line antidiabetic medications with cardiovascular events among insured adults with type 2 diabetes. JAMA Netw Open 2018; 1(8): e186125

19. Abdul-Ghani M, Jayyous A, Asaad N, Helmy S and Al-Suwaidi J. Pioglitazone and cardiovascular risk in T2DM patients: is it good for all? Ann Trans/ Med 2018; 6(10): 192

20. Pioglitazone - European Medicines Agency summary of product characteristics. Accessed on March 2019

21. Liao HW, Saver JL, Wu YL, Chen TH, Lee M et al. Pioglitazone and cardiovascular outcomes in patients with insulin resistance, pre-diabetes and type 2 diabetes: a systematic review and meta-analysis. BMJ Open 2017; 7: e013927

22. Qin $Y$, Wang $X$, Zhang M. Effect of thiazolidinediones on renal outcomes in diabetic patients with microalbuminuria or macroalbuminuria - a systematic review and meta-analysis. Diabetes 2018; 67(1)

23. Sitagliptin - European Medicines Agency summary of product characteristics. Accessed on March 2019

23. Vildagliptin - European Medicines Agency summary of product characteristics. Accessed on March 2019

25. Linagliptin - European Medicines Agency summary of product characteristics. Accessed on March 2019

26. Alogliptin - European Medicines Agency summary of product characteristics. Accessed on March 2019

27. Saxagliptin - European Medicines Agency summary of product characteristics. Accessed on March 2019

28. Scheen AJ. Utilisation des antidiabétiques oraux en cas d'insuffisance rénale. Rev Med Suisse 2012; 8: 1614-1620

29. Green JB, Bethel MA, Armstrong PW, Buse JB, Engel SS et al. Effect of sitagliptin on cardiovascular outcomes in type 2 diabetes. N Engl J Med 2015; 373: 232-242

30. White WB, Cannon CP, Heller SR, Nissen SE, Bergenstalet RM al. Alogliptin after acute coronary syndrome in patients with type 2 diabetes. N Engl I Med 2013; 369: 1327-1335

31. Scirica BM, Bhatt DL, Braunwald E, Steg G, Davidsonet J et al. Saxagliptin and cardiovascular outcomes in patients with type 2 diabetes mellitus. N Eng/ J Med 2013; 369: 1317-1326

32. Rosenstock J, Perkovic V Johansen OE, Cooper ME, Kahn SE et al. Effect of linagliptin vs placebo on major cardiovascular events in adults with type 2 diabetes and high cardiovascular and renal risk - the CARMELINA randomized clinical trial. JAMA 2019; 321(1): 69-79

33. Kanasaki K. The role of renal dipeptidyl peptidase-4 in kidney disease: renal effects of dipeptidyl peptidase-4 inhibitors with a focus on linagliptin. Clin Sci (Lond) 2018; 132(4): 489-507

34. Tomovic K, Lazarevic J, Kocic G, Deljanin-llic M, Anderluh M et al. Mechanisms and pathways of anti-inflammatory activity of DPP-4 inhibitors in cardiovascular and renal protection. Med Res Rev 2019; 39(1): 404-422

35. Cornel JH, Bakris GL, Stevens SR, Alvarsson M, Bax WA et al. Effect of sitagliptin on kidney function and respective cardiovascular outcomes in type 2 diabetes: outcomes from TECOS. Diabetes Care 2016; 39(12): 2304-2310

36. Mosenzon O, Leibowitz G, Bhatt DL, Cahn A, Hirshberg B et al. Effect of saxagliptin on renal outcomes in the SAVOR-TIMI 53 trial. Diabetes Care 2017; 40: 69-76

37. Saraiva FK, Sposito AC. Cardiovascular effects of glucagon-like peptide 1 (GLP-1) receptor agonists. Cardiovasc Diabetol. 2014; 13: 142

38. Madsbad S. Review of head-to-head comparisons of glucagon-like peptide-1 receptor agonists. Diabetes, Obesity and Metabolism 2016; 18: 317-332

39. Liraglutide - European Medicines Agency summary of product characteristics. Accessed on March 2019

40. Exenatide - European Medicines Agency summary of product characteristics. Accessed on March 2019

41. Dulaglutide - European Medicines Agency summary of product characteristics. Accessed on March 2019

42. Semaglutide - European Medicines Agency summary of product characteristics. Accessed on March 2019

43. Albiglutide - European Medicines Agency summary of product characteristics. Accessed on March 2019

44. Lixisenatide - European Medicines Agency summary of product characteristics. Accessed on March 2019

45. Marso SP, Daniels GH, Brown-Frandsen K, Kristensen P, Mann JFE et al. Liraglutide and cardiovascular outcomes in type 2 diabetes. N Engl J Med 2016; 375: 311-322

46. Holman RR, Bethel MA, Mentz RJ, Thompson VP, Lokhnygina $Y$ et al. Effects of once-weekly exenatide on cardiovascular outcomes in type 2 diabetes. N Engl J Med 2017; 377: 1228-1239

47. Marso SP, Bain SC, Consoli A, Eliaschewitz FG, Jódaret E et al. Semaglutide and cardiovascular outcomes in patients with type 2 diabetes. N Eng/ J Med 2016; 375: 1834-1844

48. Hernandez AF, Green JB, Janmohamed S, D’Agostino RB, Granger CB et al. Albiglutide and cardiovascular outcomes in patients with type 2 diabetes and cardiovascular disease (Harmony Outcomes): a double-blind, randomised placebo-controlled trial. The Lancet 2018; 392(10157): 1519-1529

49. Pfeffer MA, Claggett B, Diaz R, Dickstein K, Gerstein HC et al. Lixisenatide in patients with type 2 diabetes and acute coronary syndrome. N Engl J Med 2015; 373: 2247-2257

50. Muskiet MHA, Tonneijck L, Smits MM, van Baar MJB, Kramer MHH et al. GLP-1 and the kidney: from physiology to pharmacology and outcomes in diabetes. Nat Rev Nephrol 2017; 13(10): 605-628 
51. Mann JFE, Ørsted DD, Brown-Frandsen K, Marso SP, Poulter NR et al. Liraglutide and renal outcomes in type 2 diabetes. N Engl J Med 2017; 377: 839-848

52. Bethel MA, Mentz RJ, Merrill P, Buse JB, Chan JC et al. Renal outcomes in the exenatide study of cardiovascular event lowering (EXSCEL). Diabetes 2018; 67 (1)

53. Dulaglutide demonstrates superiority in reduction of cardiovascular events for a broad range of people with type 2 diabetes. Available in: https://investor.lilly.com/news-releases/news-release-details/trulicityr-dulaglutide-demonstrates-superiority-reduction. Accessed on April 2019

54. Tuttle KR, Lakshmanan MC, Rayner B, Busch RS, Zimmermann AG et al. Dulaglutide versus insulin glargine in patients with type 2 diabetes and moderate-to-severe chronic kidney disease (AWARD-7): a multicentre, open-label, randomised trial. Lancet Diabetes Endocrinol. 2018; 6(8): 605-617

55. Chao EC and Henry RR. SGLT2 inhibition - a novel strategy for diabetes treatment. Nat Rev Drug Discov. 2010; 9(7): 551-559

56. Dapagliflozin - European Medicines Agency summary of product characteristics. Accessed on March 2019

57. Empagliflozin - European Medicines Agency summary of product characteristics. Accessed on March 2019

58. Canagliflozin - European Medicines Agency summary of product characteristics. Accessed on March 2019

59. Ertugliflozin - European Medicines Agency summary of product characteristics. Accessed on March 2019

60. Azharuddin M, Adil M, Ghosh P and Sharma M. Sodium-glucose cotransporter 2 inhibitors and fracture risk in patients with type 2 diabetes mellitus: A systematic literature review and Bayesian network meta-analysis of randomized controlled trials. Diabetes Res Clin Pract 2018; 146: 180-190
61. Ryan PB, Buse JB, Schuemie MJ, DeFalco F, Yuan Z et al. Comparative effectiveness of canagliflozin, SGLT2 inhibitors and non-SGLT2 inhibitors on the risk of hospitalization for heart failure and amputation in patients with type 2 diabetes mellitus: A real-world meta-analysis of 4 observational databases (OBSERVE-4D). Diabetes Obes Metab 2018; 20(11): 2585-2597

62. Duarte R, Melo M, Silva-Nunes J, Melo PC, Raposo JF, et al. SPD National recommendations for the treatment of hyperglycemia in type 2 diabetes - update based in the ADA/EASD joint position statement. Revista Portuguesa de Diabetes 2018; 13(4): 154-180

63. Zinman B, Wanner C, Lachin JM, Fitchett D, Bluhmki E et al. Empagliflozin, cardiovascular outcomes, and mortality in type 2 diabetes. N Engl J Med 2015; 373: 2117-2128

64. Neal B, Perkovic V, Mahaffey KW, de Zeeuw D, Fulcher G et al. Canagliflozin and cardiovascular and renal events in type 2 diabetes. N Engl J Med 2017; 377: 644-657

65. Wiviott SD, Raz I, Bonaca MP, Mosenzon O, Kato ET et al. Dapagliflozin and cardiovascular outcomes in type 2 diabetes. N Engl J Med 2019; 380: 347-357

66. Perkovic V, Jardine MJ, Neal B, Bompoint S, Heerspink HJL et al. Canagliflozin and renal outcomes in type 2 diabetes and nephropathy. N Engl J Med 2019

67. Heerspink HJL, Kosiborod M, Inzucchi SE, Cherney DZI. Renoprotective effects of sodium-glucose cotransporter-2 inhibitors. Kidney Int. 2018; 94(1): 26-39

\section{Correspondence to:}

Ana Duarte Gonçalves Ferreira, MD

Hospital Garcia de Orta, Avenida Torrado da Silva, 2805-267, Almada

E-mail: adgonfer@gmail.com 